\title{
Turbo PIC and Antenna Diversity for Wireless Block Fading DS-CDMA Communications
}

\author{
Simone Morosi, Enrico Del Re, Ottavio Gremigni \\ Dipartimento di Elettronica e Telecomunicazioni \\ University of Florence, Via S. Marta 3, Firenze, Italy \\ e-mail: \{morosi, delre, gremigni\}@lenst.det.unifi.it
}

\begin{abstract}
This paper presents a Turbo Multiuser detector for Turbo-Coded DS-CDMA systems, which is based on the utilization of Parallel Interference Cancellation scheme and antenna diversity in the receiver in a wireless rich-scattering block fading channel. Multiple Access Interference effects are naturally reduced by antenna diversity, and, successively, completely eliminated by a single turbo cancellation iteration. The proposed Turbo-PIC detector is proposed, highlighting its remarkable performance in term of BER: particularly, we will stress out the low complexity required to suppress MAI and achieve excellent results, also in the block fading environment where the high correlation value strongly limits single user bound.
\end{abstract}

\section{INTRODUCTION}

The wireless coded CDMA system are greatly impaired by the Multiple Access Interference (MAI) and the poor capacity of error correction of the turbo codes on the slowly fading channels [1]. Optimal joint decoding/detection is an excellent solution to this problem, but it is characterized by a prohibitive computational complexity for the actual implementations. In contrast, suboptimal solutions, which separate the operations of symbol detection and channel decoding, appears more attractive for the practical applications. Since the proposal of turbo codes [2], the "Turbo-Principle", i.e., the soft information iterative exchange between receiver constituent blocks, has been foreseen as a possible booster for the Multiuser Detection (MUD) schemes. The attention has been devoted to Parallel Interference Cancellation (PIC) receivers because of their relatively reduced complexity compared to the linear detectors and the small delay with respect to serial cancellation.

For what concerns wireless channel drawbacks, the performance degradation due to the MAI and to the lack of a general coordination between the users, is remarkable in richscattering block fading environments. Antenna diversity at the receiver has been proposed as a low-cost solution to the fading impairments [3]. Furthermore, spatial diversity at the BaseStation (BS) provides an increased signal-to-noise ratio, which helps to remove more accurately MAI thanks to a better initial estimate of the received signal [4].

Moreover, the recent studies on Multiple Input Multiple Output (MIMO) systems [5] [6] [7] showed that it is possible to exploit a rich scattering wireless channel to separate users

This work was supported by Italian National Research Program VICOM and, as a consequence, reduce the MAI. According to [7] a multiuser CDMA scheme with one antenna per user and antenna array at the BS can be regarded as a MIMO single user system; therefore, we can state that near-capacity-limit performance are achievable using both MUD and spatial diversity [6] [7].

The aim of this paper is to show that the utilization of low complexity iterative receiver, such as the PIC, together with receive diversity permits to completely eliminate MAI even for heavily loaded systems in highly correlated fading channels.

It is important to point out that different PIC strategies are proposed and compared herein: in the considered working conditions, a general strategy against wireless multiple access drawbacks fails and suitable specific approaches have to be defined.

It is worth stressing that the ultimate limit for the proposed scheme is the single user bound: since turbo codes fail to recover errors in presence of a strongly correlated fading channel, performance in block fading channel can be regarded as a sort of worst case bound for the wireless communications: this scenario results to be extremely important in an implementation perspective. Nonetheless a proper diversity schemes naturally leads to user separation, boosts the convergence of cancellation algorithm and affords remarkable performance to the turbo MUD receivers.

\section{System Model}

We have considered the up-link of a synchronous DSCDMA communication system, where the BPSK baseband symbol spreading is performed by a PN code. Each user utilizes a single element antenna, i.e., no transmit diversity is introduced, and transmits a coded stream to a BS with $n_{r}$ receiving antennas: the receiving antennas are properly spaced so each of them receives independent replicas of the transmitted signals.

Without loss of generality, we can assume that one generic bit-interval is observed. The baseband signal received in antenna $j$-th can be written as:

$$
r^{j}(t)=\sum_{k=1}^{K} h_{j k} c_{k} s_{k}(t)+n(t) \quad t \in\left[0, T_{b}\right]
$$

where: 
- $K$ is the number of active users;

- $T_{b}$ is the bit interval;

- $h_{j k}$ is the complex channel coefficient of the link between the antenna $j$-th and the $k$-th user;

- $c_{k} \in\{+1,-1\}$ is the coded bit transmitted by $k$ user;

- $s_{k}(t)$ is the $k$-th user's spreading sequence;

- $n(t)$ is an Additive White Gaussian Noise (AWGN) process with double sided spectrum density $\sigma^{2}=N_{o} / 2$ $[\mathrm{W} / \mathrm{Hz}]$

A Rayleigh block-fading channel model as in [5] has been assumed: $h_{j k}$ is a complex coefficient and its real and imaginary part are Gaussian distributed with zero mean and $\frac{1}{2}$ variance; moreover each link is assumed to be uncorrelated and identically distributed (i.i.d. assumption). As is known, in the Block-Fading (BF) model, the fading factors for each link remain constant for the duration of the entire transmitted frame, then assuming a new, uncorrelated set of random values for the next block.

We have also assumed single path propagation between each user and each antenna at the BS and that the power which is received for each user is equally divided between each BS antenna.

The receiver structure can be described as follows:

1 The baseband signal $r^{j}(t)$ which is received by each $j$ th antenna is filtered by a Matched Filter Bank (MFB) in order to separate users. The $i$-th user's signal at the output of the MFB on the $j$-th antenna can be written as follows:

$$
y_{i}^{j}=h_{j i} c_{i}+\sum_{k=1 k \neq i}^{K} h_{j k} \rho_{k i} c_{k}+\nu_{i}^{j},
$$

where $\nu_{i}^{j}=\int_{0}^{T_{b}} n(t) s_{i}(t) d t$ is the filtered Gaussian noise sample and $\rho_{k i}=\frac{1}{T_{b}} \int_{0}^{T_{b}} s_{i}(t) s_{k}(t) d t$ is the normalized crosscorrelation coefficient between users $k$-th and $i$-th.

2 For each user the Maximal Ratio Combining (MRC) is performed collecting signal's replicas from each antenna. At the output of the combiner the desired $i$-th user's signal is:

$$
\begin{aligned}
y_{i}^{(M R C)} & =\sum_{j=1}^{n_{r}}\left|h_{j i}\right|^{2} c_{i}+\sum_{j=1}^{n_{r}} \sum_{k=1 k \neq i}^{K} h_{j i}^{*} h_{j k} \rho_{k i} c_{k} \\
& +\sum_{j=1}^{n_{r}} h_{j i}^{*} \nu_{i}^{j} .
\end{aligned}
$$

It is worth stressing that, due to the previous hypotheses, the statistical mean $E\left[h_{j i}^{*} h_{j k}\right]$ is equal to zero if a infinite number of antenna elements are taken into account: the considered channel naturally decorrelates users if a high degree of space diversity is introduced. Nonetheless, since a limited number of antenna elements is considered, the second term in (3) is only an approximation of the crosscorrelation and, therefore, MAI is not completely eliminated after MRC blocks. In order to highlight the gain achieved by means of the antenna diversity at the
BS, we define the combined SNR per bit at the output of the MRC as follows [9]:

$$
\overline{\gamma_{b}}=n_{r} \frac{E_{b}}{N_{o}} .
$$

As it can be noted, the SNR has been increased $n_{r}$ times after MRC, so providing two benefits [4] : firstly, a better initial signal estimate has been obtained; besides, a more accurate MAI estimation can be achieved, due to the lower error rate.

3 The combined signal $y^{(M R C)}$ is fed into the TurboPIC detector which provides interference cancellation and turbo decoding. Based upon the estimated bits, MAI is generated for all users and subtracted from the received signal to get the desired signal (with a reduced MAI level) for each user. The $K$ soft outputs of PIC are the inputs for the decoders of each user. In the following section the Turbo-PIC receiver will be analyzed in more detail.

\section{The ITERATIVE TURbo-PIC ReCEIVER}

An iterative cancellator consists of an Interference Cancellation (IC) based Multiuser Detector followed by $K$ single-user turbo decoders. Each constituent block iteratively provides soft information to the others.

The PIC stage delivers soft outputs $\tilde{y}_{k}$ to the input of turbo decoders. After one decoding iteration ${ }^{1}$, the extrinsic information of coded bits are fed back to the cancellator and assumed equal to the a priori information for the next iteration.

This type of detector has been shown to achieve very good performance in AWGN and fast fading environments [10] [11]. Our effort is to investigate the possibility to use the same scheme even for BF channel by means of space processing.

The considered turbo codes are composed by two Recursive Systematic Convolutional (RSC) codes linked by an interleaver; a MAP based algorithm is used for iterative decoding. Since the IC receiver requires soft information about the reliability of both the systematic and the parity bits, the decoding algorithm is properly modified to produce also extrinsic information about the latter [10]. At each new iteration, the iterative structure permits the multiuser receiver to have a more reliable a priori information and the decoders to operate on the soft inputs, after the cancellation of a remarkable amount of interference.

In this work we used a partial PIC mainly derived by the one proposed by Divsalar et alii [12], with a slight modification in the calculation of extrinsic information, together with some other PIC receivers derived from the literature. As it is well known, the receiver proposed by Divsalar significantly reduces the effects of interference by removing only a fraction of MAI at each stage according to data estimates' reliability. As stated above, the convergence of cancellation algorithm can be boosted using extrinsic information coming from the turbo decoders; hence, the combination antenna diversity, partial PIC and turbo decoding should provide high performance in terms of MAI suppression with few turbo-MUD iterations.

\footnotetext{
${ }^{1}$ The number of turbo decoder iteration could also be greater than one.
} 


\section{A. Theorical Outline}

After MRC block, the $i$-th user's signal at the output of MRC is fed to the PIC device, which attempts to eliminate MAI using other users' signal estimates. The signal at the output of the $s$-th stage of the cancellator can be written as follows:

$$
\tilde{y}_{i}^{(s)}=\bar{p}_{i}^{(s)}\left(y_{i}^{(M R C)}-\hat{I}_{i}^{(s)}\right)+\left(1-\bar{p}_{i}^{(s)}\right) \tilde{y}_{i}^{(s-1)},
$$

where $\hat{I}_{i}^{(s)}$ is the amount of reconstructed interference and $\bar{p}_{i}^{(s)}$ is the weight for the $s$-th stage. The a posteriori LogLikelihood-Ratio (LLR) of the $i$-th user at the $s$-th stage is defined as follows:

$$
L^{(s)}\left(c_{i}\right) \triangleq \log \frac{p\left(c_{i}=+1 \mid y_{i}^{(M R C)}, \tilde{y}_{i}^{(s-1)}, \hat{I}_{i}^{(s)}\right)}{p\left(c_{i}=-1 \mid y_{i}^{(M R C)}, \tilde{y}_{i}^{(s-1)}, \hat{I}_{i}^{(s)}\right)} .
$$

By means of Bayes' rule, equation (6) can be rewritten as:

$$
\begin{aligned}
L^{(s)}\left(c_{i}\right) & =\log \frac{p\left(y_{i}^{(M R C)} \mid c_{i}=+1, \tilde{y}_{i}^{(s-1)}, \hat{I}_{i}^{(s)}\right)}{p\left(y_{i}^{(M R C)} \mid c_{i}=-1, \tilde{y}_{i}^{(s-1)}, \hat{I}_{i}^{(s)}\right)}+ \\
& +\log \frac{p\left(\tilde{y}_{i}^{(s-1)} \mid c_{i}=+1, \hat{I}_{i}^{(s)}\right)}{p\left(\tilde{y}_{i}^{(s-1)} \mid c_{i}=-1, \hat{I}_{i}^{(s)}\right)} \\
& +\log \frac{p\left(c_{i}=+1, \hat{I}_{i}^{(s)}\right)}{p\left(c_{i}=-1, \hat{I}_{i}^{(s)}\right)}
\end{aligned}
$$

Under the assumptions that users are statistically independent and terms $\tilde{y}_{i}^{(s-1)}$ and $y_{i}^{(M R C)}$ are uncorrelated, eq. (7) can be approximated by:

$$
\begin{aligned}
L^{(s)}\left(c_{i}\right) & =\log \frac{p\left(y_{i}^{(M R C)} \mid c_{i}=+1, \hat{I}_{i}^{(s)}\right)}{p\left(y_{i}^{(M R C)} \mid c_{i}=-1, \hat{I}_{i}^{(s)}\right)} \\
+ & \log \frac{p\left(\tilde{y}_{i}^{(s-1)} \mid c_{i}=+1\right)}{p\left(\tilde{y}_{i}^{(s-1)} \mid c_{i}=-1\right)}+\log \frac{P\left(c_{i}=+1\right)}{P\left(c_{i}=-1\right)} .
\end{aligned}
$$

The first and the second terms represent the extrinsic information $L_{e x}^{(s)}$ at the output of $s$-th stage, while the third one is the a priori information $L_{a p}^{(s)}$ provided by previous stages and decoders.

The tentative decision function we use takes into account both extrinsic information from the decoders and channel outputs [8]: in particular, the extrinsic information term that takes into account the channel information is [12]:

$$
L_{e x}^{(s)}=\frac{2}{\bar{p}_{i}^{(s)} \sigma_{i, s}^{2}} \tilde{y}_{i}^{(s)},
$$

where $\sigma_{i, s}^{2}$ is the noise-plus-residual interference variance and $\bar{p}_{i}^{(s)}$ is the weight coefficient at stage $s$. Soft estimates of coded bits, $L_{e x}^{(s)}$ are achieved by a suitable hyperbolic tangent tentative decision function with the a priori information coming from previous stages and decoders, as in the following :

$$
\begin{gathered}
\hat{c}_{i}^{(s)}=\tanh \left(\frac{L_{e x}^{(s)}+L_{a p}^{(s)}}{2}\right), \\
L_{a p}^{(s)}=L_{e x}^{(s-1)}+L_{e x_{d e c}} .
\end{gathered}
$$

In order to keep the required complexity low, the weights $\bar{p}_{i}^{(s)}$ are pre-assigned and noise-plus-residual interference variance $\sigma_{i, s}^{2}$ is approximated by thermal noise variance $\sigma^{2}$. At the first iteration, the parameters are set as follows:

$$
\begin{gathered}
\hat{c}_{i}^{(0)}=\operatorname{sgn}\left(y_{i}^{(M R C)}\right) \\
\tilde{y}_{i}^{(0)}=y_{i}^{(M R C)} .
\end{gathered}
$$

After last cancellation, the soft outputs are sent to the inputs of turbo decoders, which use these values in the calculation of probability transition.

\section{B. The considered PIC schemes}

In this work we have considered two different PIC schemes, both derived by the general Turbo-PIC, that has been described previously. Aiming to keep the required complexity low with no performance loss, we compared the performance results, of a Total PIC (TPIC) and a Modified Weighted PIC (MWPIC).

The TPIC is a classical one-stage cancellator where all the cancellation weights have been set equal to 1 for all the users.

Conversely, the MWPIC device has been introduced to achieve the best performance thanks to the space processing introduction. As shown in section II, antenna diversity helps to obtain a better initial estimate of the received signal; hence, the reconstructed signals can be considered more reliable and cancelled with a higher weight. The weights which are assigned to the cancellation stages are pre-calculated.

We want to highlight that in each of the iterations, both a parallel interference cancellation and complete turbo decoding operation are performed but the latter requires more additions and multiplications than the former, also introducing a considerable delay since all the frame has to be received to start the decoding: this assumption still holds if MAP decoder is elimated and sub-optimal decoding algorithms are introduced.

Moreover, we have also taken into account the possibility to increase the slope of the tentative decision so that in a second cancellation stage, a larger amount of MAI can be subtracted. The slope of the $\tanh ($ ) function is controlled by the parameter $\alpha$, which needs to be calculated [13].

\section{Simulation Results}

The effectiveness of the proposed receiver has been investigated through computer simulations. In order to mitigate the complexity burden due to the implementation of a non-linear decision device, the $\tanh ()$ function has been approximated through an eight-values look-up table.

For all the simulations we have uses a rate $R_{c}=1 / 2$ turbo code, composed by two 8-state RSC codes with generator polynomials $G_{0}=(13)_{8} G_{1}=(15)_{8}$, and the Block Interleaver recommended by the UMTS standard [14]. The BF 


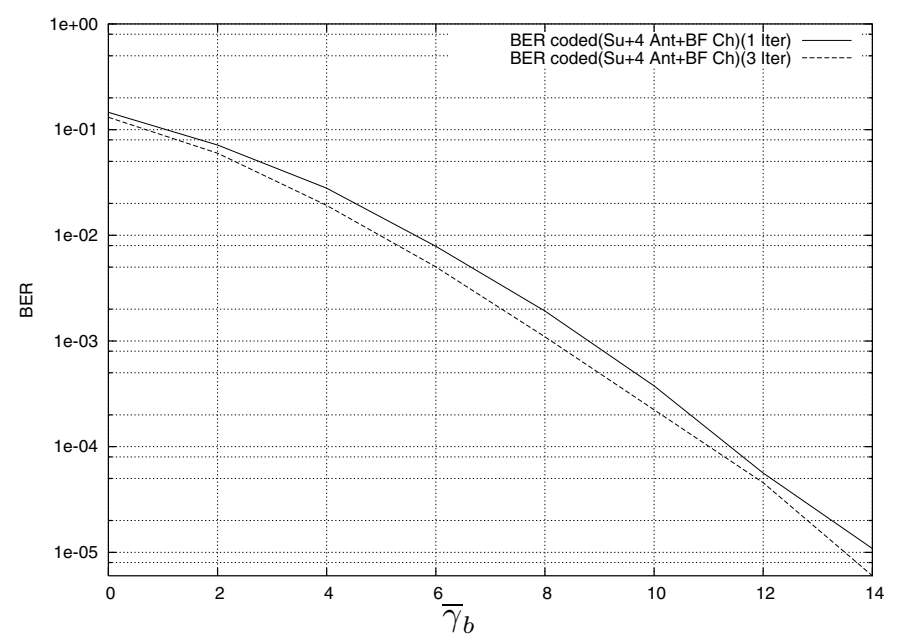

Fig. 1. BER performance for the SU case with 1 and 3 decoding iterations. Turbo Codes with rate $R_{c}=1 / 2$, number of receiving antennas $n_{r}=4$, frame length equal to 800 .

Rayleigh channel model presented in section II represents the worst operative condition for the proposed scheme [15]. We have also assumed that all the signal and channel parameters are known at the receiver whereas the carrier acquisition problem has not been addressed in the paper.

In order to show the performance degradation due to the use of turbo codes in a correlated fading channel, the single user (SU) case has been considered. We noted that the increase of the number of decoding iterations is ineffective in a correlated fading environment; in Fig. 1 it is shown that three iterations provide a slight gain $(<1 \mathrm{~dB})$ over the single iteration case and no additional gain is reached with more than three iterations. The performance of the system could be improved only by means of an external interleaver; however, this solution can result in the introduction of an intolerable decoding delay. Nevertheless antenna diversity is an essential feature to control the degradation in performance caused by fading; this effect is particularly detrimental in low-mobility scenarios, e.g., indoor wireless channels, which are well modelled by the BF channel used in this work.

In the Multiuser environment, we analyze the performance of the system with 8 active users, processing gain $G=16$ and frame length equal to 800 . The spreading sequences are PN codes with crosscorrelation factor $\rho \leq 0.75$ and the BS uses 4 separate antennas to receive users' signal. In Fig 2 we reported the performance of the conventional receiver, i.e., matched filter banks followed by turbo decoder, in the case of a SU case and 8-users systems without interference cancellation: in the multiuser case, the conventional system rapidly reaches a floor due to the MAI; the natural decorrelation effect provided by the channel is not sufficient to recover the performance loss. We could obtain better results only with a very large number of antennas; however, this solution is quite impractical. We also noted that the BER itself of the MAI-free case is limited; $\mathrm{BER}=10^{-3}$ is reached only at $\bar{\gamma}_{b}=9 \mathrm{~dB}$ : this result

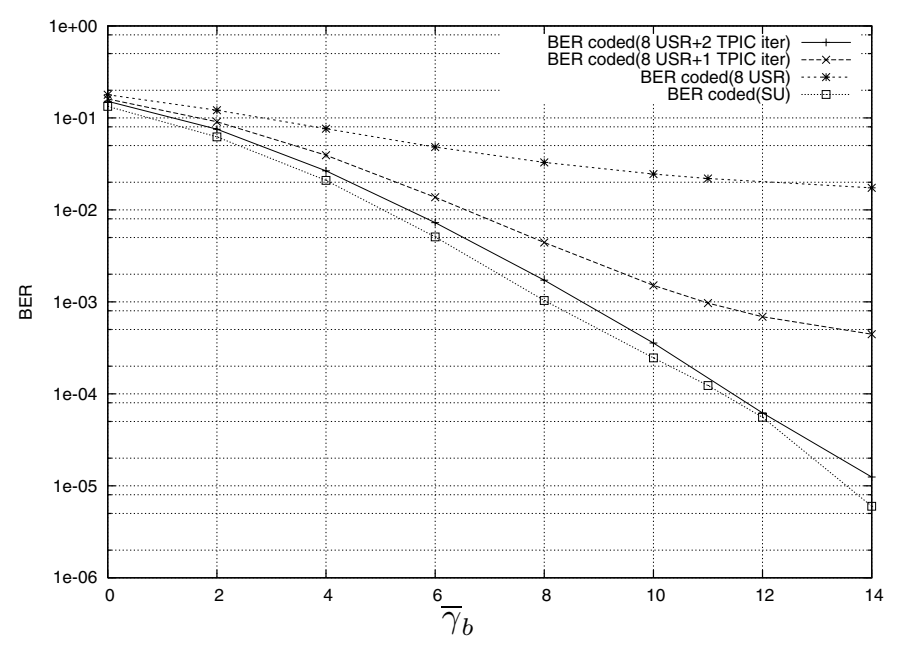

Fig. 2. BER performance of the proposed system using a TPIC device $(K=8, G=16)$. Users have equal power.

is due to the fact that the single user bound of turbo codes schemes is strongly limited by correlated fading. On the other hand, it is possible to see the performance of the proposed scheme employing a TPIC device. Two Turbo-PIC iterations are needed to reduce MAI to negligible levels and get closer than $1 \mathrm{~dB}$ to the SU bound BER.

The results, obtained by using a MWPIC with weights $p_{1}=$ $0.8, p_{2}=1.0, \alpha=4.0$, are shown in Fig. 3. The MWPIC outperforms the TPIC in BER performance and has a lower complexity because it needs just one iteration to reach the SU bound ${ }^{2}$.

In this case the slope of the hyperbolic tangent tentative decision function has been increased: this strategy implies that the device acts more like a hard decision one, so confirming the assumption made on the improved reliability due to antenna diversity.

Finally, in order to highlight the remarkable MAI suppression of the proposed schemes, we have studied an overloaded system with load factor $\beta=K / G=2.4$. We have assumed 12 active users, processing gain $G=5$ and $\rho=0.6$, the analysis has been carried out at a given $\bar{\gamma}_{b}=8 \mathrm{~dB}$. As shown in Fig. 4 , the TPIC needs 5 iterations to get very close to the MAI-free performance $^{3}$, whereas the MWPIC performance bounded is far from this limit. This behavior can be explained by noting that the MWPIC set of parameters has not been optimized for this case: if a new set is considered $\left(p_{1}=0.6, p_{2}=0.8, \alpha=\right.$ 2) MWPIC achieves better performance than TPIC with the same number of iterations (Fig. 5).

\section{CONCLUSION}

In this paper a Turbo-PIC detector based on receive diversity has been presented. The utilization of antenna diversity provides better signal's estimate at the receiver and helps mitigate

\footnotetext{
${ }^{2}$ Since the main part of the complexity burden is due to the turbo decoding, to reduce the number of turbo iteration is quite beneficial.

${ }^{3}$ This bound represents the asymptotic value for the SU case.
} 


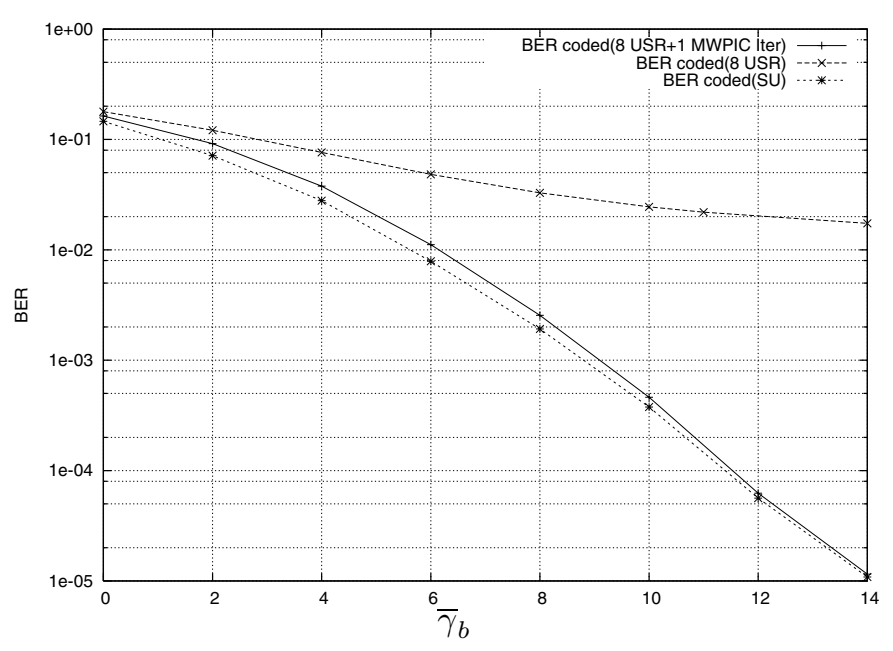

Fig. 3. BER Performance of the MWPIC ( $K=8, G=16)$. Users have equal power.

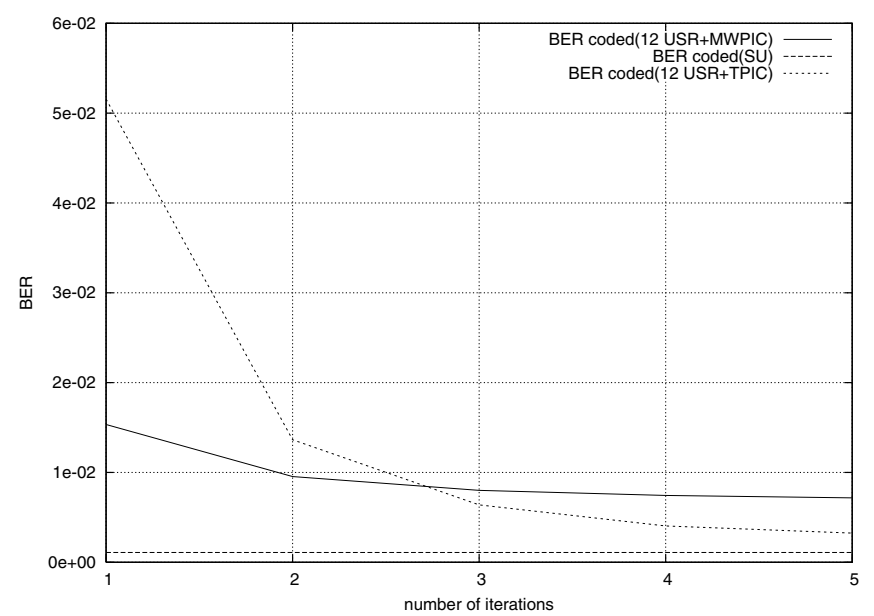

Fig. 4. BER versus the number af iterations for the TPIC and MWPIC $\left(\bar{\gamma}_{b}=8 \mathrm{~dB}\right)$. Overloaded system with $\beta=2.4$.

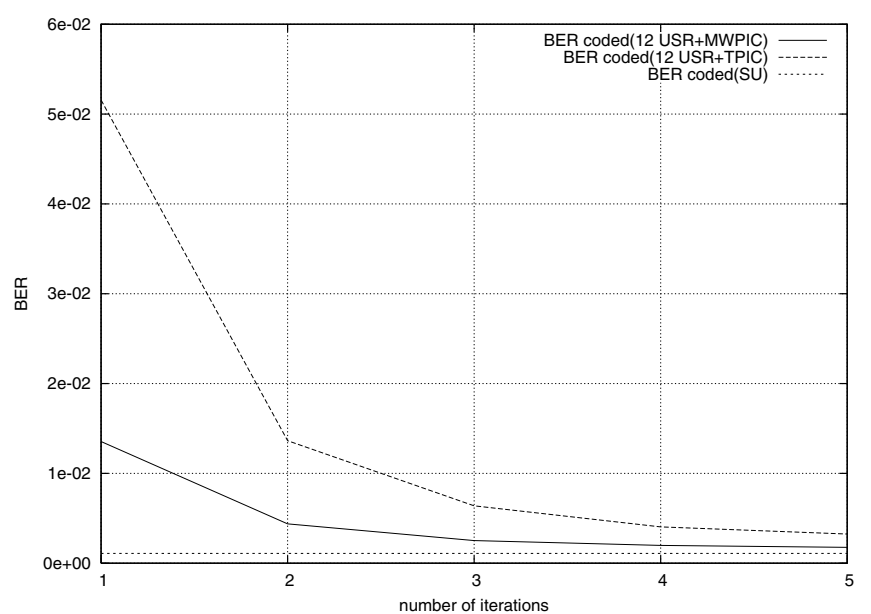

Fig. 5. Performance of the TPIC and MWPIC (with a new set of parameters) against iterations at $\bar{\gamma}_{b}=8 \mathrm{~dB}$. Overloaded system with $\beta=2.4$. the loss in performance due to fading. The results obtained for the proposed scheme show that interference can be almost completely eliminated, even in the case of strongly correlated channel, such as the BF model used.

\section{REFERENCES}

[1] M. Valenti and B. Woerner, "Performance of Turbo Codes in Interleaved Flat Fading Channels with Estimated Channel State Informaton", in Vehicular Technology Conference, Vol. 1, pp. 160-174, 1998.

[2] C. Berrou, A. Glavieux and P. Thitimajshima, "Near Shannon limit errorcorrecting coding and decoding: Turbo codes", in Proc. 1993 Int. Conf. on Communications (ICC'93), Washington DC, USA, pp. 34, June 2001.

[3] R. D. Gitlin, J. Saltz, and J. H. Winter, "The Impact of Antenna Diversity on the Capacity of Wireless Communication systems", IEEE Transactions on Communications, Vol. 42, No.4, pp. 1740-1751, 1994.

[4] N. Kong, "Space-Time Multistage Parallel Interference Cancellation (MPIC) for CDMA", in Vehicular Technology Conference, pp. 28262833, 2000.

[5] G. Foschini and M. Gans, "On Limits of Wireless Communication in a Fading Environmentwhen using Multiple Antennas", in Wireless Personal Communication, Kluver Academic Publishers, pp. 311-335, 1998.

[6] W. Rhee, W. Yu and J. Cioffi, "Utilizing Multiuser Diversity for Multiple Antenna Systems", in Wireless Communication and Networking Conference, Vol. 1, pp. 420-425, 2000.

[7] W. Rhee and J. Cioffi, "Ergodic Capacity of Multi-Antenna Gaussian Multiple-Access Channels", in Conference Record of the Thirty-Fifth Asilomar Conference on Signals, Systems and Computers, Vol. 1, pp. 507512, 2001.

[8] S. Morosi, E. Del Re, R. Fantacci and A. Bernacchioni,'”Improved Iterative Parallel Iterference Cancellation Receiver for DS-CDMA 3G Systems", in Proc. of the IEEE Wireless Communications and Networking Conference (WCNC 2003), Vol. 2, pp. 877-882, March 2003.

[9] J. G. Proakis, Digital Communications, 3rd ed. New York: McGraw-Hill, 1995.

[10] X. Wang and H. V. Poor, "Iterative (Turbo) Soft Interference Cancellation and Decoding for coded CDMA", IEEE Transactions on Communications, Vol. 47, No.7, pp. 1046-1061, July 1999.

[11] K.-M. Wu and C.-L. Wang, "An Iterative Multiuser receiver Using Partial Parallel Interference Cancellation for Turbo-Coded DS-CDMA Systems", in Proc. of GLOBECOM 2001, S.Antonio, TX, USA, pp. 244248, November 2001.

[12] D. Divsalar, M. K. Simon and D. Raphaeli, "Improved Parallel Interference Cancellation for CDMA", IEEE Transactions on Communications, Vol. 46, No.2, pp. 258-268, February 1998.

[13] B. Cho, D. Choi, S. Lee and Y. Oh, "Performance of the Improved PIC Receiver for DS-CDMA over Rayleigh Fading Channels", in Proc. of ISSSTA, pp. 45-49, Sept. 2000.

[14] 3GPP, TR 25.221 multiplexing and channel coding, Tech. Rep., 3GPP, 2001.

[15] A. Burr and J. Shen, "A Turbo-PIC Multiuser Receiver with Receive Diversity for CDMA Systems", in COST 273, May 2002 